This item was submitted to Loughborough's Research Repository by the author.

Items in Figshare are protected by copyright, with all rights reserved, unless otherwise indicated.

\title{
'Why don't you try it again?' A comparison of parent led, home based interventions aimed at increasing children's consumption of a disliked vegetable
}

\section{PLEASE CITE THE PUBLISHED VERSION}

http://dx.doi.org/10.1016/j.appet.2014.12.216

\section{PUBLISHER}

(C) Elsevier Ltd.

\section{VERSION}

AM (Accepted Manuscript)

\section{PUBLISHER STATEMENT}

This work is made available according to the conditions of the Creative Commons Attribution-NonCommercialNoDerivatives 4.0 International (CC BY-NC-ND 4.0) licence. Full details of this licence are available at: https://creativecommons.org/licenses/by-nc-nd/4.0/

\section{LICENCE}

CC BY-NC-ND 4.0

\section{REPOSITORY RECORD}

Holley, Clare, Emma Haycraft, and Claire V. Farrow. 2014. "'why Don't You Try It Again?' A Comparison of Parent Led, Home Based Interventions Aimed at Increasing Children's Consumption of a Disliked Vegetable". Loughborough University. https://hdl.handle.net/2134/16683. 
1

2

3

4

5

6

7

8

9

10

11

12

13

14

15

16

17

18

19

20

21 Running head: Increasing consumption of a disliked vegetable

Clare E Holley ${ }^{1}$

Emma Haycraft ${ }^{1}$

Claire Farrow ${ }^{*}$

${ }^{1}$ Loughborough University, Leicestershire, UK

${ }^{2}$ Aston University, Birmingham, UK Health Sciences, Aston University, Aston Triangle, Birmingham B4 7ET. Tel

+44(0)1212045384. Email c.farrow@aston.ac.uk.

20

* Address correspondence or requests for reprints to: Dr. Claire Farrow, School of Life \& 
Abstract

23 Previous research suggests that the use of modelling and non-food rewards may be effective at increasing tasting, and consequential liking and acceptance, of a previously disliked food. Although successful school-based interventions have been developed, there is a lack of research into home-based interventions using these methods. This study aimed to develop and investigate the efficacy of a parent led home-based intervention for increasing children's acceptance of a disliked vegetable. A total of 115 children aged 2-4 years were allocated to one of four intervention groups or to a no-treatment control. The four intervention conditions were: repeated exposure; modelling and repeated exposure; rewards and repeated exposure; or modelling, rewards and repeated exposure. Children in all of the intervention conditions were exposed by a parent to daily offerings of a disliked vegetable for 14 days. Liking and consumption of the vegetable were measured pre and post-intervention. Significant increases in post-intervention consumption were seen in the modelling, rewards and repeated exposure condition and the rewards and repeated exposure condition, compared to the control group. Significant post-intervention differences in liking were also found between the experimental groups. Liking was highest (>60\%) in the modelling, rewards and repeated exposure group and the rewards and repeated exposure group, intermediate $(>26 \%)$ in the modelling and repeated exposure and repeated exposure groups, and lowest in the control group (10\%). Parent led interventions based around modelling and offering incentives may present cost efficient ways to increase children's vegetable consumption.

Key words: role modelling; non-food rewards; repeated exposure; vegetable; parent led; intervention 
'Why don't you try it again?' A comparison of parent led, home based interventions aimed at increasing children's consumption of a disliked vegetable

Childhood obesity is one of the biggest public health challenges of the 21 st century, with more than 40 million children under the age of five being overweight or obese globally (World Health Organisation, 2014). As part of a healthy lifestyle, adequate vegetable consumption is known to provide numerous benefits including preventing obesity and chronic disease (Heidemann et al., 2008; Maynard, Gunnell, Emmett, Frankel, \& Davey Smith, 2003; Vioque, Weinbrenner, Castelló, Asensio, \& Garcia de la Hera, 2008). However, many adults and children are failing to consume the recommended UK quota of five portions of fruit and vegetables a day (e.g., Guenther, Dodd, Reedy, \& Krebs-Smith, 2006; Lennox, Olson, \& Gay, 2011). Given that eating behaviours track through childhood into adulthood (e.g., Lytle, Seifert, Greenstein, \& McGovern, 2000; Mikkilä, Räsänen, Raitakari, Pietinen, \& Viikari, 2007), effective interventions aimed at increasing vegetable consumption early in childhood are required.

The development of liking and acceptance of foods is influenced by numerous factors, such as how palatable foods are, their nutritional content and their associated emotional experience (e.g. party or reward foods versus everyday foods) (e.g., Birch, Zimmerman, \& Hind, 1980; Mikula, 1989; Mobini, Chambers, \& Yeomans, 2007; Steiner, 1979). One theory behind acquisition of liking and acceptance of foods is 'learned safety', where repeated ingestion of an unfamiliar food without negative gastro-intestinal consequences leads to increased acceptance of that food (Kalat \& Rozin, 1973).

Furthermore, if positive consequences are experienced (such as satiety), preference may develop for that food (Kalat \& Rozin, 1973). In this way, repeated exposure can be used to transform disliked or unfamiliar foods into accepted (Pliner \& Loewen, 1997) or even liked (Lakkakula, Geaghan, Zanovec, Pierce, \& Tuuri, 2010) foods. Previous research suggests that in order to increase liking of novel foods in two year olds, between five and 10 exposures may be necessary (Birch, Birch, Marlin, \& Kramer, 1982; Birch, Gunder, Grimm- 
Tomas, Laing, \& Grimm-Thomas, 1998), while 15 exposures may be required to increase preferences among 3-4 year olds (Sullivan \& Birch, 1990). Vegetables are commonly disliked by children (e.g., Cashdan, 1998; Skinner, Carruth, Bounds, \& Ziegler, 2002) and a body of evidence supports the use of repeated exposure to increase children's liking of vegetables (e.g., Ahern, Caton, Blundell, \& Hetherington, 2014; Caton et al., 2013; Hausner, Olsen, \& Møller, 2012; Wardle, Herrera, Cooke, \& Gibson, 2003; Wardle et al., 2003). Although this is promising evidence for the use of repeated exposure to transform children's dislike of vegetables, persuading children to repeatedly try previously rejected vegetables may prove difficult. Indeed, many parents do not continue to expose children to foods once they have been rejected (Birch, McPhee, Shoba, Pirok, \& Steinberg, 1987), where the number of exposures necessary to alter a child's preferences is more than parents offer. Combining other methods with repeated exposure may help to encourage parents to repeatedly offer, in turn improving children's liking and acceptance of vegetables. With this in mind, it would be valuable to explore techniques which may be used alongside repeated exposure to facilitate tasting and improve the likelihood of increasing children's intake of previously refused vegetables.

One technique that could be used alongside repeated exposure is modelling. Modelling occurs through a process of observational learning, where encouragement and facilitation of behaviours results in them becoming habitual (Bandura, 1977). Peer modelling of eating behaviour has been shown to be effective at increasing children's acceptance of novel healthy foods (Hendy, 2002) as well as altering children's food choices (Birch, 1980). Parental modelling of healthy eating has also been associated with children's subsequent consumption of fruits and vegetables (Draxten, Fulkerson, Friend, Flattum, \& Schow, 2014; Gregory, Paxton, \& Brozovic, 2010; Palfreyman, Haycraft, \& Meyer, 2012). Parental modelling has been shown to significantly increase children's willingness to try an unfamiliar food compared to when children were simply offered the unfamiliar food (Harper \& Sanders, 1975), suggesting that parental modelling could indeed be a successful method for increasing children's willingness to taste novel or disliked foods. 
In addition, the use of contingent non-food rewards may be another strategy which can be used to aid children's liking of new or previously refused foods. One contingent reward or incentive that is often used with young children is a sticker. The use of stickers as rewards has been shown to be successful at increasing consumption of healthy snack foods in eight children aged between three and six (Stark, Collins, Osnes, \& Stokes, 1986).

Furthermore, non-food rewards have proved to be a successful component of repeated exposure interventions aimed at increasing children's consumption of disliked or novel vegetables in both the school (Añez, Remington, Wardle, \& Cooke, 2013; Cooke et al., 2011; Hendy, Williams, \& Camise, 2005) and home environments (Corsini, Slater, Harrison, Cooke, \& Cox, 2013; Remington, Anez, Croker, Wardle, \& Cooke, 2012). Although these programmes generally describe the rewards given as tangible rewards (e.g., stickers or a small toy), such reward systems inevitably have a social reward element entrenched within them (i.e. praise).

Previous research has investigated the use of these techniques (repeated exposure, modelling and non-food rewards) in combination to increase children's liking and consumption of vegetables. Interventions using these techniques within a school-based setting have already generated successful results. For example, the Bangor Food Research Unit's ‘Food Dudes' programme (Lowe, Dowey, Horne, \& Murcott, 1998), which combines peer modelling, rewards and exposure, has been rolled out in schools across the UK and Ireland. Although successful at increasing children's liking and consumption of vegetables in the short term (e.g., Horne, Lowe, Bowdery, \& Egerton, 1998; Horne et al., 2011; Lowe et al., 1998; Lowe, Horne, Tapper, Bowdery, \& Egerton, 2004; Tapper, Horne, \& Lowe, 2003), the 'Food Dudes' and other similar programmes rely on local government funding and whole school sign-up, making such programmes inaccessible for many families. Home-based parent led interventions provide an alternative to such programmes (Fildes, van Jaarsveld, Wardle, \& Cooke, 2013). Similar research about parent led interventions in the home setting has been conducted (e.g., Añez et al., 2013; Corsini et al., 2013; Remington et al., 2012), and these studies suggest that repeated exposures incentivised with rewards can be 
effective at increasing children's consumption of a disliked vegetable. The current study builds on this research by further investigating whether parental modelling can be used to increase children's liking and acceptance, and how this may interact with rewards.

The present study concerns a home-based intervention, grounded in the principles of rewards, modelling and repeated exposure. It aimed to evaluate the intervention's success at increasing children's liking and consumption of a previously disliked vegetable. Four intervention conditions were tested. All of these conditions used repeated exposure, with one testing the effect of just repeated exposure (condition 1), one testing modelling paired with repeated exposure (condition 2), one testing rewards paired with repeated exposure (condition 3), and one comprising all of these methods (modelling, rewards and repeated exposure-condition 4). The fifth condition was a no-treatment control group (condition 5). It was predicted that children who participated in the all methods condition (comprising modelling, rewards and repeated exposure; 4) would show significant increases in both liking and consumption of a previously disliked target vegetable post-intervention when compared to the control group (5). It was further predicted that increases in liking and consumption of the target vegetable would be intermediate for children in the modelling and repeated exposure condition (2), and the rewards and repeated exposure condition (3) and smallest in the repeated exposure condition (1) relative to the control group (5).

\section{Method}

\section{Participants}

One hundred and thirty six parent-child pairs were recruited to take part in this study. Children were aged from 25 to 55 months ( $M=38$ months; SD = 7.75 months). This age group was selected as fussy eating and neophobia (avoidance of new foods) are commonly seen around this age (Addessi, Galloway, Visalberghi, \& Birch, 2005) and during this preschool period when children typically spend more time with their parents it may be easier for parents to deliver a home-based intervention. 


\section{Procedure}

Full ethical clearance for this study was obtained from Loughborough University's Institutional Review Board. Informed consent was obtained from all parents before the onset of the study, with parents fully advised of their right to withdraw themselves and their child at any point.

Recruitment

Parents were recruited via 20 parent and toddler groups and childcare centres in the

East Midlands, UK. Following approval from the manager or group leader, mutually convenient times were agreed for testing to take place. Parents were approached by the researcher and invited to participate in a home-based study investigating methods which parents can use to help their children eat vegetables. Parents who expressed an interest in participating were then given an information sheet detailing the study before providing consent for their own and their child's participation, with participation limited to one child per family. Parents were not compensated for their participation in this study.

Target vegetables

In line with previous research (e.g. Remington et al., 2012), each child was assigned a single target disliked vegetable. Assigning just one target vegetable also helped to keep the intervention simple and minimised the chances of the participants being overwhelmed or put-off by the intervention. Parents were asked to rank a list of six raw vegetables (baby corn, celery, red pepper, cherry tomato, cucumber, and sugar snap peas) in order of their own preference, with 1 being the one they liked best and 6 being the one they liked least. Parents were told that if they did not know whether their child liked the vegetable (as the vegetable was not familiar to the child) they should not rank the vegetable. This allowed disliked vegetables to be assigned rather than novel ones. These six vegetables were chosen as the research team deemed them to be commonly consumed by adults, readily 
available, being simple to prepare, and keeping in the fridge for a number of days without spoiling (thereby minimising waste). Parents were then asked to repeat this process according to their child's preferences. The vegetable ranked fourth for the child was allocated as the target vegetable for the intervention, avoiding those ranked fifth or sixth to allow for both positive and negative shifts in liking (Cooke et al., 2011). Because some conditions required parents to model eating the vegetable, if the child's fourth ranked vegetable was ranked as fifth or sixth by parents, an alternative disliked vegetable was selected to limit any confounding effects of parental preferences. Children's dislike of the target vegetable was confirmed during a baseline session with the researcher (see Baseline section below).

All target vegetables were presented at baseline and post intervention in their raw form, washed, chopped into approximately $2.5 \mathrm{~g}$ pieces (which were small enough to fit in the mouth) and served in $30 \mathrm{~g}$ portions, weighed using Salter dietary electronic scales 1250 . This weight was chosen as it represents more than an age-appropriate portion for children in this age group (NHS Choices, 2009; Infant \& Toddler Forum, 2013), thereby reducing the possibility that any child would choose to eat the entire portion.

\section{Baseline}

During a baseline session, parent-child dyads were each tested separately from other dyads. Parents were asked to provide demographic information for themselves and their child including age, ethnicity, number of children and their highest level of education.

\section{Measures}

Children's liking of the target vegetable was measured using a 3-point smiley face scale (Birch, Zimmerman, \& Hind, 1980) which comprises three stylised, gender neutral faces. One with a broad smile to represent 'yummy, I like it!', one neutral to represent 'ok' and one with a down-turned mouth to represent 'yucky, I don't like it!'. The smiley faces rating scale is seen as a more reliable measure of liking than pure verbalisations in children 
of this age (Blissett, Haycraft, \& Farrow, 2010; Weisberg \& Beck, 2010). Children were

214 familiarised with this scale at a baseline session.

\section{Familiarisation}

Children were shown a brief child-friendly information sheet, which largely comprised pictures, to familiarise them with the protocol of the session and the researcher talked to them about what would be involved. Children were also familiarised with the 3-point smiley faces scale. Each face was explained to them (with a description of how each of the faces would reflect how much they liked a food) and their ability to correctly identify the expression of each face's was verified in a procedure similar to Weisberg \& Beck (2010). Here, each child was asked to correctly identify which face represented "yucky", "yummy" or "just ok". Next, children were shown and asked to name the target vegetable which had been assigned to them, with it presented in its whole form. Children who could not name the vegetable were told its name and the vegetable was placed on the table in front of them.

Testing baseline consumption and liking

Children were then given a small plastic pot containing $30 \mathrm{~g}$ of their target vegetable. The vegetable had been chopped into child-sized pieces $(\sim 2.5 \mathrm{~g})$. The children were asked to remove the lid of the pot and tell the researcher what was inside. Again, children who could not name the chopped vegetable were told its name. This process was chosen to ensure that the children linked the chopped vegetable to what it looks like in its whole form, aiming to minimise the effects of how the vegetable was later presented by parents. Children were then asked to try a piece of the target vegetable. If reluctant, children were gently encouraged by the researcher to first choose a piece to pick up with their fingers, then to lick the piece and, if possible, to progress to biting or eating the piece. Children were not encouraged to swallow the piece, so as to avoid causing stress to the children, and in an effort to increase their willingness to try the vegetable. Whether or not each child tasted the 
vegetable (defined as licking, sucking, biting or chewing) was then recorded by the researcher.

Once the children had tried the vegetable (or after they had refused to try it) they were asked "Do you like [name of vegetable]?". They were then asked to rate their liking using the 3-point smiley faces scale ('yummy', 'ok' or 'yucky'). Children were then told that they could eat as much as they wanted of the vegetable in the pot, and a free eating session commenced. This session lasted a maximum of five minutes or was terminated when the children said that they did not want any more or when they left the test table. The test portion of the target vegetable was then removed and re-weighed (including pieces which were tasted but not consumed - i.e. licked or chewed but rejected) in order to measure consumption.

Intervention groups and allocation

Recruitment centre groups were systematically assigned by the primary investigator to one of four experimental conditions: 1. repeated exposure; 2. modelling and repeated exposure; 3. rewards and repeated exposure; or 4. modelling, rewards and repeated exposure. This method of allocation was chosen to prevent discussion of the study methods between parents in different intervention groups. Consecutive sampling was used, so that a maximum number of dyads could be recruited from each centre. Centres were sequentially allocated to each condition. If there was not space in the next condition in the sequence, the centre was pragmatically assigned to an alternative condition, creating even sized conditions. Parents in all of these conditions were instructed to offer their child a small piece ( 2.5g, which they were shown an example of during the baseline session) of the target vegetable (which was provided for parents by the research team) each day for 14 consecutive days, using the protocol for the intervention condition to which they were assigned. Parents were asked to conduct all offerings outside of a mealtime in line with previous research (Fildes et al., 2013), in order to avoid adding any potential stress associated with mealtimes. Parents in the repeated exposure condition (1) were instructed to 
simply offer their child a small piece of the target vegetable without eating it themselves. They were also asked to remain neutral in their responses to whether or not their child tasted the piece. Parents in the modelling and repeated exposure condition (2) were instructed to eat a small piece of the target vegetable in front of their child, expressing a positive response such as "oh this [name of vegetable] is really nice!". These parents were instructed to offer their child a small piece of the vegetable immediately afterwards, but to remain neutral regardless of whether their child tried a piece of the vegetable. Parents in the rewards and repeated exposure condition (3) were asked to offer their child a small piece of the target vegetable, telling them that if they try a piece they can choose a sticker from a sheet provided for the study. Parents were further told that if their child did try a piece of the vegetable, they should not only give them the sticker they chose but also praise them with a phrase such as "well done, you tried your [name of vegetable]!" and to tell their child that they were receiving a sticker because they tried the vegetable. Finally, parents in the modelling, rewards and repeated exposure condition (4) were instructed to eat a piece of the target vegetable in front of their child, saying how nice it was, and then to offer their child a piece telling them they could choose a sticker if they tried it, and giving praise if the child did indeed try a piece. Parents in all conditions were instructed to adhere to their assigned method of offering for the entire 14 day period, and to record the success of the protocol in a 'tasting diary'. This diary asked parents to record whether they completed each daily offering, and included a daily manipulation check (e.g., 'Did you stay neutral?' in the repeated exposure group) as well as a record of whether each offering resulted in a tasting (defined as contact with the child's mouth, including licking, sucking, biting and chewing, where swallowing was not necessary). During the baseline session, the researcher verbally explained to parents how to offer the vegetable and how to use the diary, and written instructions on how to complete the daily offerings were also provided. Parents were also given the opportunity to ask any questions about the protocol, and given the researcher's contact information should they have any further queries. 
Fourteen day follow-up consumption and liking

After the 14 day intervention period, parent-child dyads attended a follow-up session at the toddler group they attended at baseline. This session was identical in format to the baseline session, in order to allow for comparison of liking and consumption of the target vegetable pre and post-intervention. Parent and child height $(\mathrm{cm})$ and weight $(\mathrm{kg}$; using Salter 9059 SS3R ultra-slim scales) were measured. Parents also returned their completed tasting diaries.

\section{Data analysis}

Sample size was calculated following Cohen's (1992) guidelines of adequate sample size for statistical power. Based on these guidelines, a minimum of 16 dyads in each condition was required in order to detect a small effect with power of 0.8 and $p<.05$. To account for attrition across the study, participants were over-recruited by fifty percent, meaning that a minimum of eight additional dyads were recruited to each condition. For detailed information about attrition per condition please see Figure 1. Child height and weight were converted into age and gender adjusted BMI z scores (Cole, Freeman, \& Preece, 1995; Freeman et al., 1995). Exploratory analyses were conducted to check normality of the data. Parent BMI and child age and the total tastings achieved were nonnormally distributed. Consumption data both pre and post were also non-normally distributed, with a floor effect of a large number of zero scores. For these reasons, data were analysed using non-parametric tests where possible and parametric tests (ANOVAs) were conducted where there was no suitable alternative. Repeated measures ANOVAs were used to assess whether there were significant differences in any changes in consumption between the groups across the intervention period. Kruskal-Wallis analyses were conducted to investigate any potential differences between group consumption pre-intervention, consumption post-intervention, and the total tastings achieved. Mann-Whitney $U$ analyses were then used to compare each experimental group's target vegetable consumption to that of the control group and the total tastings achieved between experimental groups. This 
324 allowed for assessment of whether, post-intervention, participants in each condition

325 consumed significantly more in comparison to the control group. Finally, chi-square analyses

326 were used to look for differences in liking of the target vegetable between groups, both pre

327 and post-intervention.

\section{Results}

\section{Sample and attrition}

Of the 136 participants who completed the baseline session, 21 families (14.8\%)

332 were unavailable for the 14 day follow-up or withdrew from the study (due to illness, work

333 commitments, or other personal reasons), leaving a sample of 115 parent-child dyads. Of

334 these participants, 98 parents identified themselves as White/Caucasian, six identified as

335 Black/Black British, two identified as Asian/Asian British and nine parents did not provide this

336 information. The flow of participants through the study is shown in Figure 1. Based on

337 previous research suggesting that 10 tastings of a disliked food are necessary for children to 338 acquire liking (Sullivan \& Birch, 1990), all analyses were repeated for a subset of the sample

339 whose tasting diaries indicated that they had achieved 10 or more offerings (and removing 340 those classed as 'non-completers' who achieved fewer than 10 offerings). However, as the

341 findings of these analyses were unchanged from those using the full sample, full sample 342 analyses are reported. 


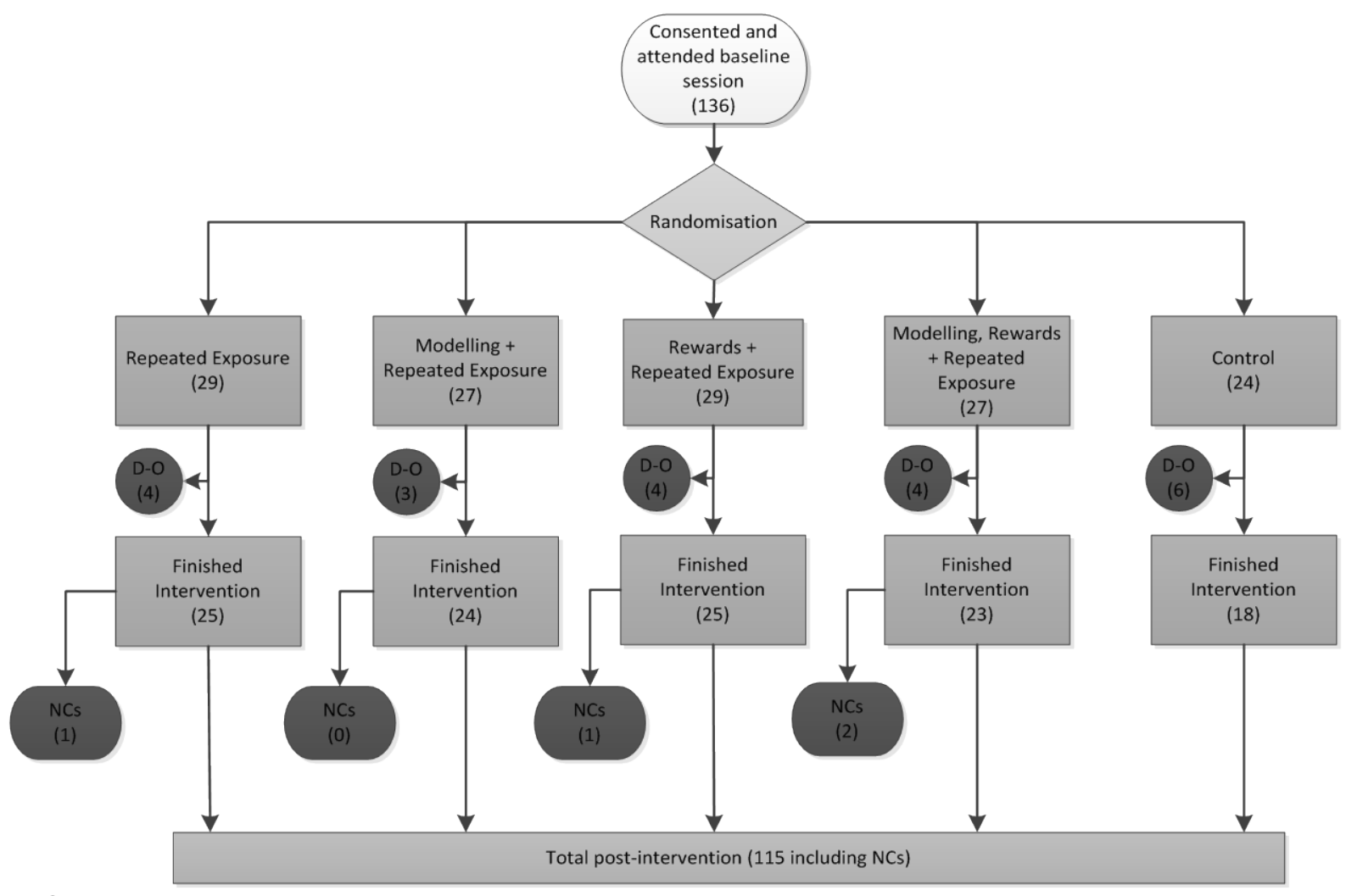

345 NCs: Non-completers - i.e. those children who received fewer than 10 offerings of the target

346 vegetable during the 14 day intervention period

347 Figure 1: Flow of parent-child dyads from baseline to post-intervention during a vegetable 348 intervention for each of five experimental conditions.

\section{Descriptive statistics}

All groups were compared for differences in child and parent characteristics,

352 including age, gender, parental education, and BMI. There were no significant differences were found for these characteristics between groups and this information is displayed in

354 Table 1. 
Table 1: Child and parent characteristics of the final sample by experimental group, and Chi-square/ANOVA tests of difference between

\begin{tabular}{|c|c|c|c|c|c|c|}
\hline & $\begin{array}{c}\text { Repeated } \\
\text { Exposure (1) }\end{array}$ & $\begin{array}{c}\text { Modelling + } \\
\text { Repeated } \\
\text { Exposure (2) }\end{array}$ & $\begin{array}{c}\text { Rewards + } \\
\text { Repeated } \\
\text { Exposure (3) }\end{array}$ & $\begin{array}{c}\text { Modelling, Rewards } \\
+ \text { Repeated } \\
\text { Exposure (4) }\end{array}$ & Control (5) & $\begin{array}{c}\text { Group } \\
\text { difference }\end{array}$ \\
\hline \multicolumn{7}{|l|}{ Parent } \\
\hline Parent Age [Years] & $34.15(4.74)$ & $35.97(5.11)$ & $35.93(5.71)$ & $36.49(3.64)$ & $32.81(4.03)$ & $F=2.15$, n.s. \\
\hline Parent BMI & $25.5(5.04)$ & $26.03(5.18)$ & $25.43(3.83)$ & $25.59(5.03)$ & $22.72(2.57)$ & $F=.58$ n.s. \\
\hline Education Level [n (\%)] & & & & & & $X^{2}=2.88$ n.s. \\
\hline Non-University graduate & $14(61)$ & $12(55)$ & $10(42)$ & $9(43)$ & $9(60)$ & \\
\hline University level or higher & $9(39)$ & $10(45)$ & $14(58)$ & $12(57)$ & $6(40)$ & \\
\hline \multicolumn{7}{|l|}{ Child } \\
\hline Child Age [Months] & $38.24(8.82)$ & $39.68(9.01)$ & $40.20(6.58)$ & $38.09(8.16)$ & $34.17(6.17)$ & $F=.14$ n.s. \\
\hline Child BMI Z score & $0.29(1.04)$ & $0.27(.77)$ & $0.07(.81)$ & $0.19(1.01)$ & $0.50(.58)$ & $F=.46$ n.s. \\
\hline Child Gender [ $n(\%)]$ & & & & & & $X^{2}=.99$ n.s. \\
\hline Male & $11(46)$ & $10(42)$ & $9(38)$ & $8(38)$ & $6(33)$ & \\
\hline Female & $13(54)$ & $14(58)$ & $15(63)$ & $13(62)$ & $12(67)$ & \\
\hline
\end{tabular}

357 Note: Mean (SD) displayed unless otherwise stated. Descriptive statistics are based on available data, with missing data in some categories. 
359

\section{Exploring differences among intervention and control conditions on children's} consumption of a disliked vegetable

In order to examine group differences in consumption of the target vegetable across the study, repeated measures ANOVAs were conducted. Consumption of the target vegetable significantly increased over the intervention period in all groups, with a main effect of time $(F(1,110)=25.80, p<.001)$. However, there was not a significant group by time interaction $(F(4,110)=.89, \mathrm{p}=.48)$. Pre and post-intervention consumption data per experimental group can be seen in Table 2 .

Table 2: Mean pre and post-intervention consumption of the target vegetable (in grams) per intervention condition, including minimum and maximum values with significant group differences indicated.

\begin{tabular}{|c|c|c|c|c|c|}
\hline \multirow[t]{2}{*}{ Intervention condition } & \multicolumn{3}{|c|}{ Pre Consumption } & \multicolumn{2}{|c|}{ Post Consumption } \\
\hline & $\mathrm{N}$ & $\begin{array}{l}\text { Mean (g) } \\
(\mathrm{SD})\end{array}$ & Min / Max & $\begin{array}{c}\text { Mean }(g) \\
(\mathrm{SD})\end{array}$ & Min / Max \\
\hline Repeated Exposure (1) & 25 & $\begin{array}{l}0.28 \\
(0.78)\end{array}$ & $0.00 / 3.60$ & $\begin{array}{l}2.90 \\
(5.30)\end{array}$ & $0.00 / 19.35$ \\
\hline $\begin{array}{c}\text { Modelling + Repeated } \\
\text { Exposure (2) }\end{array}$ & 24 & $\begin{array}{l}0.36 \\
(0.60)\end{array}$ & $0.00 / 2.00$ & $\begin{array}{r}4.68 \\
(8.37)\end{array}$ & $0.00 / 30.00$ \\
\hline $\begin{array}{c}\text { Rewards + Repeated } \\
\text { Exposure (3) }\end{array}$ & 25 & $\begin{array}{l}0.48 \\
(0.87)\end{array}$ & $0.00 / 2.50$ & $\begin{array}{l}3.65^{a} \\
(6.83)\end{array}$ & $0.00 / 30.00$ \\
\hline $\begin{array}{l}\text { Modelling, Rewards + } \\
\text { Repeated Exposure (4) }\end{array}$ & 23 & $\begin{array}{l}0.61 \\
(1.06)\end{array}$ & $0.00 / 3.40$ & $\begin{array}{l}3.96^{\mathrm{b}} \\
(5.64)\end{array}$ & $0.00 / 22.15$ \\
\hline Control (5) & 18 & $\begin{array}{l}0.25 \\
(0.54)\end{array}$ & $0.00 / 2.15$ & $\begin{array}{l}1.14^{a, b} \\
(1.92)\end{array}$ & $0.00 / 5.85$ \\
\hline
\end{tabular}

${ }^{a}$ Significant difference in post-intervention consumption between groups 3 and $5(p<.05)$ 
Kruskal-Wallis analyses revealed that pre-intervention, there were no significant differences between the groups on children's consumption of the target vegetable $(H(4)=$ 3.29, $p=.51)$. A series of Mann-Whitney $U$ tests revealed that pre-intervention there were no significant differences in consumption of the target vegetable between any pairings of the five groups. There were also no significant differences between the groups on children's consumption of the target vegetable post-intervention $(H(4)=5.07, p=.28)$. However, Mann-Whitney $U$ tests revealed that post-intervention, consumption was significantly higher for children in the modelling, rewards and repeated exposure group (4) $(\mathrm{Mdn}=1.65, \mathrm{U}=$ 137.00, $z=-1.98, p=.02, r=-.31)$, and the rewards and repeated exposure group (3) (Mdn $=50, \mathrm{U}=155.00, z=-1.82, \mathrm{p}=.03, r=-.28)$ compared to the control group $(\mathrm{Mdn}=.00)$. No significant differences were observed in post-intervention consumption amongst the modelling and repeated exposure (2) (Mdn $=.00, \mathrm{U}=176.00, z=-1.14, \mathrm{p}=.13, r=.18$ ) or the repeated exposure group (1) (Mdn $=.00, \mathrm{U}=198.00, z=-.77, \mathrm{p}=.23, r=.12)$, when compared to the control group $(\mathrm{Mdn}=.00)$.

\section{Exploring differences between the intervention conditions on the total number of tastings achieved}

Previous research has shown that children need to try disliked foods a large number of times for them to become liked (e.g., Sullivan \& Birch, 1994). With this in mind, analyses were used to explore whether there were significant differences in the number of tastings achieved between the intervention groups. Tasting data were the total number of reported tastings from the parent diaries. Kruskal-Wallis analysis revealed that there were significant group differences in the number of tastings achieved across the intervention period $(H(3)=$ 15.53, $p=.001)$. A series of Mann-Whitney $U$ tests revealed that the number of tastings achieved was significantly higher in the modelling, rewards and repeated exposure group (4) $(\mathrm{Mdn}=12.00, \mathrm{U}=116.50, \mathrm{z}=-2.63, \mathrm{p}=.004, \mathrm{r}=-.06)$ and rewards and repeated exposure group (3) $(\mathrm{Mdn}=11.00, \mathrm{U}=137.50, z=-2.61, p=.004, r=-.06)$ compared to the repeated exposure group $(1)(M d n=6.00)$. The modelling, rewards and repeated exposure group (4) 
$402(\mathrm{Mdn}=12.00, \mathrm{U}=105.50, \mathrm{z}=-2.90, \mathrm{p}=.002, \mathrm{r}=-.07)$ and rewards and repeated exposure 403 group (3) $(\mathrm{Mdn}=11.00, \mathrm{U}=125.00, \mathrm{z}=-2.90, \mathrm{p}=.002, \mathrm{r}=-.06)$ also achieved significantly 404 more tastings than the modelling group (2) $(\mathrm{Mdn}=5.00)$. There were no significant 405 differences in the number of tastings achieved between the modelling, rewards, and

406

407

408

409

410

411

412

413

414

415

416

417

418

419

420

421

422

423 repeated exposure group $(4)(\mathrm{Mdn}=12.00, \mathrm{U}=229.00, \mathrm{z}=-.53, \mathrm{p}=.30, \mathrm{r}=-.01)$ and the rewards group $(3)(\mathrm{Mdn}=11.00)$, or between the modelling and repeated exposure group (2) (Mdn $=5.00, U=220.50, z=.00, p=.50, r=.00)$ and the repeated exposure group (1) $(\mathrm{Mdn}=6.00)$.

\section{Exploring differences among the intervention and control conditions on children's}

\section{liking of a previously disliked vegetable}

Of the 115 children who took part in the study, 39 did not appear to fully understand the smiley faces rating scale which was used to determine children's opinion of the target vegetable. These children could not correctly identify the "yummy" or "yucky" faces on request. Children who could not use the smiley faces rating scale were removed from the liking analyses, although it is noted that this resulted in uneven group sizes. The number of children able to use the smiley faces rating scale can be seen in Table 3 , alongside the percentages of children within each condition who rated the target vegetable as "yummy" both pre and post-intervention.

Table 3: Number of children rating the target vegetable as "yummy" on the smiley faces rating scale pre and post-intervention per condition

\begin{tabular}{lccc}
\hline Experimental Group & N & Yummy Pre & Yummy Post \\
\hline Repeated Exposure (1) & 20 & 0 & 7 \\
Modelling + Repeated Exposure (2) & 15 & 0 & 4 \\
Rewards + Repeated Exposure (3) & 16 & $1^{*}$ & 10 \\
Modelling, Rewards + Repeated Exposure (4) & 15 & $2^{*}$ & 9
\end{tabular}


* Children were only assigned this vegetable when they rated it as yummy but then only ate one small piece of it or less - i.e. where their response was considered incongruent with their true liking. difference in rated liking between the five groups $\left(x^{2}(8, N=76)=11.52, p=.16, V=.28\right)$. However, post-intervention there was a significant difference between the groups on children's rated liking of the target vegetable $\left(x^{2}(8, N=76)=15.48, p=.05, V=.32\right)$. Here, the proportion of children who rated the target vegetable as "yummy" was highest in the modelling, rewards and repeated exposure (4) and rewards and repeated exposure (3) groups (over 60\%), intermediate in the modelling and repeated exposure (2) and repeated exposure (1) groups (over 26\%), and lowest in the control group (5) (10\%). For exact numbers of children who rated the vegetable as "yummy" refer to table 3.

The aim of this study was to assess the effectiveness of a home-based rewards, modelling and repeated exposure intervention for increasing children's liking and acceptance of a disliked vegetable. It was predicted that children who participated in the all methods condition (4) would show significant post-intervention increases in both liking and consumption of a previously disliked target vegetable, compared to the control group (5). It was further predicted that there would be intermediate increases in liking and consumption of the target vegetable for children who were in the modelling and repeated exposure condition(2), or the rewards and repeated exposure condition (3). Finally, it was predicted that children in the repeated exposure group (1) would have the smallest post-intervention increases in liking or consumption of the target vegetable, in comparison to the control group (5). These hypotheses were partially supported. 
In the current study, post-intervention consumption and liking of the previously disliked vegetable was significantly greater amongst children who were in the all methods condition (4) than the control group (5), suggesting that a combination of parental modelling, rewards and repeated exposure is effective at increasing children's consumption and liking of a previously disliked vegetable. This is consistent with previous research using mixed methods interventions, such as the 'Food Dudes' (Horne et al., 2011; Lowe et al., 1998, 2004) and the 'Kids Choice' (Hendy et al., 2005) programmes. The current study adds to the results of these school-based interventions by suggesting that, alongside rewards, parental modelling could be an effective alternative to the peer modelling component of these interventions. It also suggests that the home environment can be a suitable setting for such interventions.

Greater consumption and liking of the disliked vegetable post-intervention was found amongst children who were in the rewards and repeated exposure condition (3), as well as the modelling, rewards and repeated exposure condition (4) when compared to those in the control group (5). Moreover, the number of tastings achieved by the intervention groups fitted the same pattern as was found for increases in liking and consumption. Specifically, the all methods group (4) and the rewards and repeated exposure group (3) achieved approximately twice as many tastes as children in the modelling and repeated exposure (2) or repeated exposure alone (1) groups. Taste exposures are likely to be necessary for a young child to accept and acquire a liking for novel or disliked foods (Birch et al., 1987), and the combination of rewards and repeated exposure appears to be most effective at increasing such tasting and subsequent consumption in this study. This finding is in line with previous research suggesting that small tangible rewards can be effective when combined with repeated exposure in both the school (Wardle et al., 2003) and home settings (Fildes et al., 2013; Remington et al., 2012). Although this appears to contradict the over-justification hypothesis of rewards (Deci, Koestner, \& Ryan, 1999), where giving rewards in exchange for consumption decreases liking for that food, it does support the current literature to date on rewarding tasting disliked compared to liked foods. As Cooke, Chambers, Añez, and Wardle 
478 (2011) discuss, rewarding children for consuming large amounts of already liked foods may 479 actually lower the intrinsic value attributed to such foods. However, if foods are not already 480 liked, then pairing such foods with a reward can result in increased liking via a process of 481 paired conditioning.

The current study found no significant differences in consumption or liking of the disliked vegetable post-intervention between children in the modelling and repeated exposure condition (2) when compared to those in the control group. This suggests that the combination of modelling and repeated exposure alone, without rewards, may not be effective at increasing liking or consumption of a previously disliked food. Although previous research suggests that enthusiastic parental modelling can be a useful tool for increasing vegetable consumption in children (e.g., Gregory et al., 2010; Harper \& Sanders, 1975; Palfreyman et al., 2012; Pearson, Biddle, \& Gorely, 2009; Tibbs et al., 2001), to our knowledge there are currently no successful interventions which use parental modelling. It is possible that previous research showing modelling to be effective has had subtle elements of rewards within the design, such as praise for tasting. In an effort to unpack the effects of rewards and modelling, parents in the current study's modelling and repeated exposure condition (2) were asked to enthusiastically model tasting of the food but were explicitly asked to remain neutral regardless of whether their child tried the vegetable (i.e. not to praise their child). Whilst previous research suggests that modelling is a relatively commonly used practice (with approximately one third of parents in Musher-Eizenman and Holub's 2007 study); this may have resulted in the parents' modelling being unnatural, where they were focused on remaining neutral or following the study instructions. It is also possible that children in this condition found it strange that they were not praised for trying a food their parent was enthusiastic about eating, as praise is thought to be a fairly common feeding practice (with $30 \%$ of parents in Orrell-Valente et al.,'s 2007 study using praise). This in turn may have reduced these children's enjoyment and subsequent liking of the vegetable. Moreover, although parents were given instructions on how to model appropriately, they may not have been sufficiently enthusiastic (see Hendy \& Raudenbush, 2000) or their enthusiasm 
may not have lasted for the duration of the intervention, thereby potentially reducing the effectiveness of their efforts.

No significant differences in post-intervention liking or consumption of the target vegetable were found between the repeated exposure group (1) and the control group. It is likely that this is because children in the repeated exposure alone group did not achieve the 10-15 tastings necessary to increase liking and consumption of the target vegetable (Birch et al., 1982; Sullivan \& Birch, 1990). Although repeated taste exposures are vital to encourage children to taste disliked foods, repeatedly offering in a neutral way did not appear to ensure tastings in this study. These findings suggest that additional methods are necessary to achieve the taste exposures needed to induce liking and acceptance of a disliked vegetable.

Overall, this study has made a valuable contribution to the knowledgebase about successful methods which can be used to encourage children to eat, and like, more vegetables. By gathering data concerning tasting, liking and consumption and including a control group as well as a repeated exposure group, we are able to build on previous research (e.g., Lowe et al., 2004; Remington et al., 2012) to compare the effects of each component of the intervention. Nevertheless, the study does have limitations. Firstly, this study sample has limited ethnic diversity, which must be considered. Due to the parent led nature of the study we were unable to fully control parents' reactions when offering the vegetable or their response to children tasting. While this means that fidelity to the intervention cannot be guaranteed for all participants, this is a wholly necessary part of developing a home-based intervention which results in high ecological validity. We also do not know whether parents offered the target vegetable at other times during the intervention, and future studies should aim to control for this. It is also important to acknowledge that some children ate the disliked food at baseline, however these children were only assigned the vegetable as their target vegetable if they ate a very small quantity, such as only the first piece they were asked to try. It is also possible that some of the target vegetables which were assigned were not strictly disliked, and may have in fact been novel, although this was controlled for wherever possible with information from parents. 
$534 \quad$ These findings indicate that parent led home-based interventions comprised of

535 repeated exposure and rewards, with or without the addition of parental modelling, are

536 successful at increasing children's consumption and liking of a previously disliked vegetable.

537 These results also suggest that in home-based interventions, neither parental modelling nor

538 repeated exposure are sufficient for increasing children's liking and consumption of a

539 disliked vegetable without the use of rewards. Although this finding is contrary to what was

540 initially expected, it could be promising that parental modelling is not vital to increase liking

541 and consumption, especially for parents who do not eat vegetables themselves or do not

542 often eat meals with their child. Such interventions have minimal economic burden and may

543 prove to be a viable alternative to school programmes which tend to be costly and exclusive.

544 Further research is required to identify whether increases in liking and consumption of a

545 previously disliked vegetable are maintained over time. 
Addessi, E., Galloway, A. T., Visalberghi, E., \& Birch, L. L. (2005). Specific social influences on the acceptance of novel foods in 2-5-year-old children. Appetite, 45(3), 264-271.

Ahern, S. M., Caton, S. J., Blundell, P., \& Hetherington, M. M. (2014). The root of the problem: increasing root vegetable intake in preschool children by repeated exposure and flavour flavour learning. Appetite, 80, 154-60.

Añez, E., Remington, A., Wardle, J., \& Cooke, L. (2013). The impact of instrumental feeding on children's responses to taste exposure. Journal of Human Nutrition and Dietetics : The Official Journal of the British Dietetic Association, 26(5), 415-20.

Bandura, A. (1977). Self-efficacy: toward a unifying theory of behavioral change. Psychological Review, 84(2), 191-215.

Birch, L. L. (1980). Effects of peer models' food choices and eating behaviors on preschoolers' food preferences. Child Development, 51(2), 489-496.

Birch, L. L., Birch, D., Marlin, D. W., \& Kramer, L. (1982). Effects of instrumental consumption on children's food preference. Appetite, 3(2), 125-134.

Birch, L. L., Gunder, L., Grimm-Tomas, K., Laing, D. G., \& Grimm-Thomas, K. (1998). Infants Consumption of a new food enhances acceptance of similar foods. Appetite, 30(3), 283-295.

Birch, L. L., McPhee, L., Shoba, B. C., Pirok, E., \& Steinberg, L. (1987). What kind of exposure reduces children's food neophobia?: Looking vs. tasting. Appetite, 9(3), 171178.

Birch, L. L., Zimmerman, S. I., \& Hind, H. (1980). The influence of social-affective context on the formation of children's food preferences. Child Development, 51(3), 856-861.

Blissett, J., Haycraft, E., \& Farrow, C. (2010). Inducing preschool children's emotional eating: relations with parental feeding practices. The American Journal of Clinical Nutrition, 92(2), 359-365.

Cashdan, E. (1998). Adaptiveness of food learning and food aversions in children. Social Science Information, 37(4), 613-632.

Caton, S. J., Ahern, S. M., Remy, E., Nicklaus, S., Blundell, P., \& Hetherington, M. M. (2013). Repetition counts: repeated exposure increases intake of a novel vegetable in UK pre-school children compared to flavour-flavour and flavour-nutrient learning. The British journal of nutrition, 109(11), 2089-2097.

Choices, N. (n.d.). 5 A DAY portion sizes - Live Well - NHS Choices. Department of Health. Retrieved June 06, 2014, from http://www.nhs.uk/Livewell/5ADAY/Pages/Portionsizes.aspx

Cohen, J. (1992). A power primer. Psychological Bulletin, 112(1), 155-9. 
Cole, T. J., Freeman, J. V, \& Preece, M. A. (1995). Body mass index reference curves for the UK, 1990. Archives of Disease in Childhood, 73(1), 25-9.

Cooke, L. J., Chambers, L. C., Añez, E. V, Croker, H. A., Boniface, D., Yeomans, M. R., \& Wardle, J. (2011). Eating for Pleasure or Profit The Effect of Incentives on Children's Enjoyment of Vegetables. Psychological Science, 22(2), 190-196.

Corsini, N., Slater, A., Harrison, A., Cooke, L., \& Cox, D. N. (2013). Rewards can be used effectively with repeated exposure to increase liking of vegetables in 4-6-year-old children. Public Health Nutrition, 16(5), 942-51.

Deci, E. L., Koestner, R., \& Ryan, R. M. (1999). A meta-analytic review of experiments examining the effects of extrinsic rewards on intrinsic motivation. Psychological Bulletin, 125(6), 627.

Draxten, M., Fulkerson, J. A., Friend, S., Flattum, C. F., \& Schow, R. (2014). Parental role modeling of fruits and vegetables at meals and snacks is associated with children's adequate consumption. Appetite, 78, 1-7.

Fildes, A., van Jaarsveld, C. H. M., Wardle, J., \& Cooke, L. (2013). Parent-Administered Exposure to Increase Children's Vegetable Acceptance: A Randomized Controlled Trial. Journal of the Academy of Nutrition and Dietetics, 114(6), 1-8.

Freeman, J. V, Cole, T. J., Chinn, S., Jones, P. R., White, E. M., \& Preece, M. A. (1995). Cross sectional stature and weight reference curves for the UK, 1990. Archives of Disease in Childhood, 73(1), 17-24.

Gregory, J. E., Paxton, S. J., \& Brozovic, A. M. (2010). Maternal feeding practices, child eating behaviour and body mass index in preschool-aged children: a prospective analysis. The International Journal of Behavioral Nutrition and Physical Activity, 7(3), 55-64.

Guenther, P. M., Dodd, K. W., Reedy, J., \& Krebs-Smith, S. M. (2006). Most Americans eat much less than recommended amounts of fruits and vegetables. Journal of the American Dietetic Association, 106(9), 1371-1379.

Harper, L. V, \& Sanders, K. M. (1975). The effect of adults' eating on young children's acceptance of unfamiliar foods. Journal of Experimental Child Psychology, 20(2), 206214.

Hausner, H., Olsen, A., \& Møller, P. (2012). Mere exposure and flavour-flavour learning increase 2-3 year-old children's acceptance of a novel vegetable. Appetite, 58(3), 1152-1159.

Heidemann, C., Schulze, M. B., Franco, O. H., van Dam, R. M., Mantzoros, C. S., \& Hu, F. B. (2008). Dietary patterns and risk of mortality from cardiovascular disease, cancer, and all causes in a prospective cohort of women. Circulation, 118(3), 230-237.

Hendy, H. M. (2002). Effectiveness of trained peer models to encourage food acceptance in preschool children. Appetite, 39(3), 217-225.

Hendy, H. M., Williams, K. E., \& Camise, T. S. (2005). "Kids Choice" school lunch program increases children's fruit and vegetable acceptance. Appetite, 45(3), 250-263. 
Horne, P. J., Greenhalgh, J., Erjavec, M., Lowe, C. F., Viktor, S., \& Whitaker, C. J. (2011). Increasing pre-school children's consumption of fruit and vegetables. A modelling and rewards intervention. Appetite, 56(2), 375-85.

Horne, P. J., Lowe, C. F., Bowdery, M., \& Egerton, C. (1998). The way to healthy eating for children. British Food Journal, 100(3), 133-140.

Infant and Toddler Forum. (2013). Combining food for a balanced diet: Fruit and vegetables. Retrieved April 23, 2014, from www.infantandtoddlerforum.org/c/document_library/get_file?uuid=ef63213d-0cdb-4d74979a-f847710f1673\&groupld=11528

Kalat, J. W., \& Rozin, P. (1973). "'Learned safety"” as a mechanism in long-delay taste aversion learning in rats. . Journal of Comparative and Physiological Psychology, 83(19), 8-207.

Lakkakula, A., Geaghan, J., Zanovec, M., Pierce, S., \& Tuuri, G. (2010). Repeated taste exposure increases liking for vegetables by low-income elementary school children. Appetite, 55(2), 226-31.

Lennox, A., Olson, A., \& Gay, C. (2011). National diet and nutrition survey. Headline results from Years. Retrieved from http://www.foodafactoflife.org.uk/attachments/8921d124960e-4f68d4b026f4.pdf

Lowe, C. F., Dowey, A. J., Horne, P. J., \& Murcott, A. (1998). Changing what children eat. In (Ed) (57-80). In A. Murcott (Ed.), "The Nation"s Diet': The social science of food choice (pp. 57-80). London: Longman,.

Lowe, C. F., Horne, P. J., Tapper, K., Bowdery, M., \& Egerton, C. (2004). Effects of a peer modelling and rewards-based intervention to increase fruit and vegetable consumption in children. European Journal of Clinical Nutrition, 58(3), 510-522.

Lytle, L. A., Seifert, S., Greenstein, J., \& McGovern, P. (2000). How Do Children's Eating Patterns and Food Choices Change Over Time? Results from a Cohort Study. American Journal of Health Promotion, 14(4), 222-228.

Maynard, M., Gunnell, D., Emmett, P., Frankel, S., \& Davey Smith, G. (2003). Fruit, vegetables, and antioxidants in childhood and risk of adult cancer: the Boyd Orr cohort. Journal of Epidemiology and Community Health, 57(3), 218-225.

Mikkilä, V., Räsänen, L., Raitakari, O. T., Pietinen, P., \& Viikari, J. (2007). Consistent dietary patterns identified from childhood to adulthood: The Cardiovascular Risk in Young Finns Study. British Journal of Nutrition, 93(06), 923-931.

Mikula, G. (1989). Influencing food preferences of children by "if European Journal of Social Psychology, 19(January), 225-241.

Mobini, S., Chambers, L. C., \& Yeomans, M. R. (2007). Effects of hunger state on flavour pleasantness conditioning at home: flavour-nutrient learning vs. flavour-flavour learning. Appetite, 48(1), 20-8. doi:10.1016/j.appet.2006.05.017 
Orrell-Valente, J. K., Hill, L. G., Brechwald, W. A., Dodge, K. A., Pettit, G. S., \& Bates, J. E. (2007). "Just three more bites": an observational analysis of parents' socialization of children's eating at mealtime. Appetite, 48(1), 37-45.

Palfreyman, Z., Haycraft, E., \& Meyer, C. (2012). Development of the Parental Modelling of Eating Behaviours Scale (PARM): links with food intake among children and their mothers. Maternal \& Child Nutrition, 1-13.

Pearson, N., Biddle, S. J. H., \& Gorely, T. (2009). Family correlates of fruit and vegetable consumption in children and adolescents: a systematic review. Public Health Nutrition, 12(2), 267-83.

Pliner, P., \& Loewen, E. R. (1997). Temperament and food neophobia in children and their mothers. Appetite, 28(3), 239-254.

Remington, A., Anez, E., Croker, H., Wardle, J., \& Cooke, L. (2012). Increasing food acceptance in the home setting: a randomized controlled trial of parent-administered taste exposure with incentives. The American Journal of Clinical Nutrition, 95(1), 72-77.

Skinner, J. D., Carruth, B. R., Bounds, W., \& Ziegler, P. J. (2002). Children's food preferences: a longitudinal analysis. Journal of the American Dietetic Association, 102(11), 1638-1647.

Stark, L. J., Collins, F. L., Osnes, P. G., \& Stokes, T. F. (1986). Using reinforcement and cueing to increase healthy snack food choices in preschoolers. Journal of Applied Behavior Analysis, 19(4), 367-379.

Steiner, J. E. (1979). Facial expressions of the neonate infant indicating the hedonics of food related stimuli. In J. M. Weiffenbach (Ed.), Taste and development: the genesis of sweet preferences (pp. 173-189). Washington DC: US department of health and health sciences.

Sullivan, S. A., \& Birch, L. L. (1990). Pass the sugar, pass the salt: Experience dictates preference. Developmental Psychology, 26(4), 546-551.

Sullivan, S. A., \& Birch, L. L. (1994). Infant dietary experience and acceptance of solid foods. Pediatrics, 93(2), 271-277.

Tapper, K., Horne, P. J., \& Lowe, C. F. (2003). The Food Dudes to the rescue. The Psychologist, 16(1), 18-21.

Tibbs, T., Haire-Joshu, D., Schechtman, K. B., Brownson, R. C., Nanney, M. S., Houston, C., \& Auslander, W. (2001). The relationship between parental modeling, eating patterns, and dietary intake among African-American parents. Journal of the American Dietetic Association, 101(5), 535-541.

Vioque, J., Weinbrenner, T., Castelló, A., Asensio, L., \& Garcia de la Hera, M. (2008). Intake of fruits and vegetables in relation to 10-year weight gain among Spanish adults. Obesity, 16(3), 664-70.

Wardle, J., Cooke, L. J., Gibson, E. ., Sapochnik, M., Sheiham, A., \& Lawson, M. (2003). Increasing children's acceptance of vegetables; a randomized trial of parent-led exposure. Appetite, 40(2), 155-162. 
Wardle, J., Herrera, M.-L. L., Cooke, L., \& Gibson, E. L. (2003). Modifying children's food preferences: the effects of exposure and reward on acceptance of an unfamiliar vegetable. European Journal of Clinical Nutrition, 57(2), 341-348.

Weisberg, D.P., Beck, S. R. (2010). Children's thinking about their own and others' regret and relief. Journal of Experimental Child Psychology, 106, 184-191.

World Health Organisation. (2014). WHO | Obesity and overweight. World Health Organization. Retrieved September 17, 2014, from http://www.who.int/mediacentre/factsheets/fs311/en/ 